\title{
Sechzehn Jahre Schriftleitung der CHIRURGISCHEN GASTROENTEROLOGIE
}

\author{
J. Horn, München
}

Es gibt Grund, die Aufmerksamkeit auf Herrn Karl-Joseph Paquet zu lenken. Der Anlaß ist sein Ausscheiden aus dem Amt des Hauptschriftleiters, aus der gestaltenden und lenkenden Arbeit an dem medizinischen Fachjournal CHIRURGISCHE GASTROENTEROLOGIE.

1984 war er zusammen mit C. E. Zöckler und H. Denck Initiator und Wegbereiter dieser deutschsprachigen Quartalszeitschrift. Das Anliegen dieser Zeitschrift war stets geteilt; es suchte in komplimentärer Vereinbarung die Bewußtmachung der sich immer mehr herauskristallisierenden Fachlichkeit der gastroenterologischen Chirurgie und die integrative Zusammenführung von fachspezifischem Wissen in ein interdisziplinäres, tragfähiges Medizinverständnis.

Das Besondere hat stets seine Bewährungsprobe im Allgemeinen, so wie das Allgemeine ohne die Aufwendungen im Besonderen unwissend und verführerisch banal bleibt. Zum Führungsstab dieser von der Idee und der Ausführung her beachtenswerten Zeitschrift gehörte wenige Monate später auch das medizinische und ärztliche Urgestein A. Akovbiantz, so daß sich Idee und Verwirklichung dieses Journals auf vier markante Pfeiler beziehen konnte, die alle gleichermaßen zum Gelingen dieses Vorhabens, das Besondere mit dem Allgemeinen zu vereinen, beteiligt waren.

Was aber heißt gelingen? Ist das Gelingen meßbar an der Höhe der Auflage, an der Zahl der Abonnenten, an verkaufstechnischen Daten, oder zeigt sich dieses Gelingen in der Treue zur Idee, in der Kontinuität ihrer Umsetzung, in der Bestätigung durch eine engagierte und dankbare Leserschaft. Es ist den Initiatoren dieser Zeitschrift, nicht weniger aber der inzwischen wechselnden Verlagsverantwortlichkeit (seit 1992: Karger-Verlag) hoch anzurechnen, sich gegen den Trend der heute üblichen Quantifizierung behauptet zu haben. Die CHIRURGISCHE GASTROENTEROLOGIE hat sich mit ihrer thematischen Schwerpunktbildung und ihrem breiten interdisziplinären Spektrum einen fest zugeordneten Stellenwert in der kaum mehr überblickbaren fachorientierten Medienvielfalt gesichert.

Wenn von solchem Gelingen die Rede ist, dann muß von K.-J. Paquet gesprochen werden, der sich von Gründungszeiten an bis zu seinem jetzigen Ausscheiden die Idee dieser Zeitschrift in unvergleichlicher Weise zu eigen gemacht hat. K.-J. Paquet repräsentiert

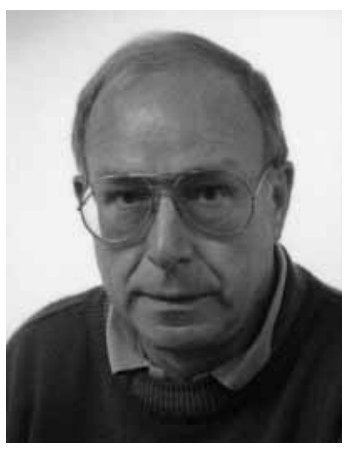

Prof. Dr. K.-J. Paquet

wie kaum ein anderer die Grundbedingungen von fachlicher Spezialisierung und interdisziplinärer Integration. Nicht Lippenbekenntnisse, sondern überzeugte und beständige Annäherung an die Maximen der Ärztlichkeit!

Nach dem Medizinstudium, in Köln, Berlin und Bonn (welches begleitet war vom Studium der Psychologie) folgten 3 Jahre fachlicher Erkundung und medizinischer Selbstfindung. Wir finden ihn in der Abteilung für Anästhesiologie des Universitätsklinikums Bonn, in der Geburtshilflich-Gynäkologischen Abteilung des Marienkrankenhauses Siegen, in der Abteilung für Innere Medizin des Barbara-Hospitals Gladbach und in der Abteilung für Angiologie und Kardiologie der medizinischen Universitätsklinik Heidelberg. Dies belegt seine Absicht, sich nicht in die Nische einer fachlichen Ausschließlichkeit drängen und devitalisieren zu lassen; immer ist er auf der Suche nach Horizonten, nie begnügt er sich mit dem Studium einzelner Lichtreflexe.

1967 beginnt er seine chirurgische Ausbildung bei Prof. Dr. Dr. A. Gütgemann an der Chirurgischen Universitätsklinik Bonn. 1971 wird Paquet Arzt für Chirurgie. 1972 schließt er seine Habilitationsarbeit ab. 1975 anvanciert er zum leitenden Oberarzt an der Chirurgischen Universitätsklinik Bonn. 1977 wird ihm die außerplanmäßige Professur zugesprochen. 1979 Arzt für Gefäßchirurgie, 1980 Arzt für Unfallchirurgie. 1981 wird Paquet Chefarzt des neu gegründeten Departements für Chirurgie und Gefäßchirurgie des Heinz Kalk-Krankenhauses, Bad Kissingen.

\section{KARGER}

Fax +497614520714

E-mail Information

www.karger.com
(C) 2000 S. Karger GmbH, Freiburg

Accessible online at:

www.karger.com/journals/cga 
$\mathrm{Zu}$ Recht wird man eine solche berufliche Karriere mit dem Prädikat «erfolgreich» apostrophieren können; damit aber wäre nichts ausgesagt über die treibenden Kräfte, den Fleiß, die Zielstrebigkeit, das Engagement und die Begeisterung sowie die täglich neu $\mathrm{zu}$ vollziehende Entscheidung für das Wesentliche und Wichtige. Die Daten der biographischen Entwicklung sind das eine, die sich in paralleler Abfolge verändernde Medizinlandschaft das andere. Einerseits die sich immer stürmischer entwickelnde Medizintechnik, andererseits die immer intensiver werdenden anonymisierenden Bürokratismen. Hier der Erfolg, dort ein wachsendes Mißtrauen. Gegen die ärztliche Intention steht das Tarifrecht, limitierend und verfremdend. Über alles breitet sich das Fait accompli einer disziplinierenden Planwirtschaft; Funktionäre haben sich des Gesundheitswesens angenommen. Das «Einerseits» war von den Ärzten versäumt, verdrängt, fehlgedeutet oder aber auch mißbraucht worden.

Vor dem Hintergrund dieser medizingeschichtlichen Jetztzeitentwicklung ist die Biographie von Paquet zu sehen und zu verstehen. Er widmete sich engagiert seiner Kliniktätigkeit, die stets die Basis bildete auch für diejenigen Anliegen, die er über sie hinaus verfolgte. Immer wieder faßt er Wissen und Erfahrung, ebenso neue Beobachtungen und Einsichten in Form von Büchern und wissenschaftlichen Publikationen zusammen und sucht in zahlreichen Vorträgen sowohl die Diskussion als auch die kritische Auseinandersetzung mit Fachkollegen. So, wie er sich immer als Lernender begreift, liebt er es auch, sich mitzuteilen, sein Wissen zur Verfügung zu stellen.

So wird er als ein Meister auf dem Gebiet der Leberchirurgie und der Behandlung des Pfortaderhochdrucks international bekannt. Jedes Wissen beinhaltet die Möglichkeit der hilfreichen Anwendung und die Aufgabe der bewußtmachenden Verbreitung. In der Überzeugung, daß das Publizieren der Sache zu dienen, nicht der eigenen Person nützlich zu sein hat, sucht Paquet nach Möglichkeiten der Mitteilung, des Gesprächs und der fachlichen Auseinandersetzung. So wird er Mitglied in zahlreichen Fachgesellschaften, wird Ehrenmitglied der slovakischen und griechischen Chirurgengesellschaft und erhält schließlich eine Gastprofessur an der Tongji Medical University in Wuhan/China.

Nach dem Gesagten ist leicht einzusehen, daß Paquet in der Gründung einer eigenen Zeitschrift eine willkommene Gelegenheit sah, seine medizinischen Vorstellungen publizistisch umzusetzen und ihnen Gehör zu verschaffen. Das Anliegen, das aktuelle Wissen didaktisch aufzuarbeiten und zu bündeln, es gleichzeitig aber in den Kontext der interdisziplinären Gesamtsicht zu stellen, wurde von den Gründungsherausgebern vorzüglich bewältigt.

Das Detail erlangte Wichtigkeit, weil es als Teil verstanden und vertreten wurde. Verantwortung bezog sich auf das Ganze, ohne sich über das Detail hinwegzusetzen. Es wurde ein Bewußtsein vermittelt, welches, in einer außer sich geratenen Zeit von hektischen Veränderungen und partikularen Interessen, Orientierung gab und welches offen blieb für ein nüchternes Abwägen und ein konstruktives Gestalten. Nach dem beklagenswerten Tod von Akovbiantz und nach dem altersbedingten Ausscheiden von Zöckler und Denck führte Paquet die Aufgabe der Hauptschriftleitung der CHIRURGISCHEN GASTROENTEROLOGIE fort.

Es gelang ihm, die anfängliche Idee der thematischen Schwerpunktbildung und der fachlichen Breite durch Originalarbeiten und interdisziplinäre Gespräche geduldig und oftmals gegen Widerstände weiterzuverfolgen. Es gelang ihm, aufgrund seiner umfangreichen Kontakte zu medizinischer Kompetenz und Fachlichkeit immer neue Mitarbeiter und ambitionierte Themen-«Kompositeure» zu gewinnen, die Qualität und inhaltliche Aktualität der einzelnen Hefte gleichermaßen gewährleisteten. Es gelang ihm, trotz immer einschneidenderer Restriktionen Sponsoren und Reklame-interessierte Firmen zu finden, die den Bestand dieser Zeitschrift sicherstellten. Es gelang ihm schließlich, die Zahl der Abonnenten kontinuierlich zu steigern und das Ansehen dieser Zeitschrift so $\mathrm{zu}$ festigen, daß die CHIRURGISCHE GASTROENTEROLOGIE heute in dem immer vielfältiger werdenden Angebot von Fachjournalen eine allseits geschätzte Position einnimmt.

Paquet hat sich mit dieser Zeitschrift einer Idee verpflichtet, in der er immer wieder seine Art, Medizin zu verstehen und sie zu praktizieren, zum Ausdruck brachte. Stets war ihm bewußt, daß Anstrengung und Durchhaltevermögen, Fleiß und Geduld erforderlich sind, um eine solche Idee lebendig zu erhalten. Er legt nun das Amt des Hauptschriftleiters nieder, um sich einer neuen Aufgabe im Medizinischen Dienst der Krankenversicherung Niedersachens zu stellen. In einer Zeit, in der das Gesundheitswesen nach neuen Wegen sucht, in einer Zeit ungehemmter Interessenkonflikte und vordergründiger Kommerzialisierung, in einer Zeit, in der die Idee von der Solidarität neu erdacht und erarbeitet werden muß, in einer solchen Zeit tut es not, Frontarbeit zu leisten. Paquet hat die Herausforderung angenommen. Sicher fällt es ihm nicht leicht, die Hauptverantwortung gegenüber der CHIRURGISCHEN GASTROENTEROLOGIE aus der Hand zu geben. Doch die Aufgabe, der er sich nun stellt, wird ganzes von ihm fordern. Herrn Paquet ist zu danken, daß er sich über 16 Jahre dieser Zeitschrift mit ganzem persönlichem Einsatz gewidmet hat. Der Zeitschrift ist zu wünschen, daß sie sich auch weiterhin engagiert, verantwortungsbewußt und ideenreich an die Hand genommen weiß. 